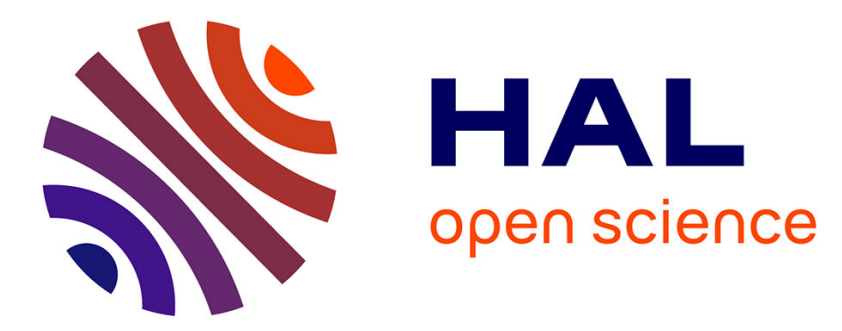

\title{
Infrared camera based on a curved retina
}

Delphine Dumas, Manuel Fendler, Frédéric Berger, Baptiste Cloix, Cyrille

Pornin, Nicolas Baier, Guillaume Druart, Jérôme Primot, Etienne Le Coarer

\section{To cite this version:}

Delphine Dumas, Manuel Fendler, Frédéric Berger, Baptiste Cloix, Cyrille Pornin, et al.. Infrared camera based on a curved retina. Optics Letters, 2015, 37 (4), pp.653-655. 10.1364/OL.37.000653 . hal-01226486

\section{HAL Id: hal-01226486 \\ https://hal.science/hal-01226486}

Submitted on 16 Jul 2021

HAL is a multi-disciplinary open access archive for the deposit and dissemination of scientific research documents, whether they are published or not. The documents may come from teaching and research institutions in France or abroad, or from public or private research centers.
L'archive ouverte pluridisciplinaire HAL, est destinée au dépôt et à la diffusion de documents scientifiques de niveau recherche, publiés ou non, émanant des établissements d'enseignement et de recherche français ou étrangers, des laboratoires publics ou privés. 


\title{
Infrared camera based on a curved retina
}

\author{
Delphine Dumas, ${ }^{1}$ Manuel Fendler,${ }^{1, *}$ Frédéric Berger, ${ }^{1}$ Baptiste Cloix,${ }^{1}$ Cyrille Pornin,,${ }^{1}$ Nicolas Baier, ${ }^{1}$ \\ Guillaume Druart, ${ }^{2}$ Jérôme Primot, ${ }^{2}$ and Etienne le Coarer ${ }^{3}$ \\ ${ }^{1}$ Commissariat à l'Energie Atomique, Léti, Minatec Campus, Optronics Department, rue des Martyrs, Grenoble, France \\ ${ }^{2}$ Office National d'Etudes et de Recherches Aérospatiales, chemin de la Hunière, Palaiseau, France \\ ${ }^{3}$ Institut de Planétologie et d'Astrophysique de Grenoble, chemin de la Piscine, Grenoble, France \\ *Corresponding author: manuel.fendler@cea.fr
}

\begin{abstract}
Design of miniature and light cameras requires an optical design breakthrough to achieve good optical performance. Solutions inspired by animals' eyes are the most promising. The curvature of the retina offers several advantages, such as uniform intensity and no field curvature, but this feature is not used. The work presented here is a solution to spherically bend monolithic IR detectors. Compared to state-of-the-art methods, a higher fill factor is obtained and the device fabrication process is not modified. We made an IR eye camera with a single lens and a curved IR bolometer. Images captured are well resolved and have good contrast, and the modulation transfer function shows better quality when comparing with planar systems.
\end{abstract}

Today, both military and civilian applications require miniaturized, light, and low-cost optical systems. To overcome these requirements, optical systems with few optics have to be found [1]. The study of vertebrate and invertebrate vision offers interesting ways to design simple optical architectures [2]. Despite the large variety of systems, animals share a common feature: their retinas rely on a curved surface. However, due to manufacturing constraints, detectors are planar. This leads to complex optical systems with multiple lenses in order to achieve a flat Petzval surface. Presently, some solutions exist to correct aberrations by a computational treatment; first results have been demonstrated in [3]. However, instead of complicating the design or adding postprocessing, a simple solution is to change our vision of the device, and more specifically the geometrical shape. By curving the focal plane array (FPA) as nature does, we are then able to exactly fit the focal surface with the detector. Subsequently, both on- and off-axis rays are well focused, the field curvature is directly suppressed, and illumination is increased off-axis [4]. In this way, bioinspiration achieves a simplification and a miniaturization of the optical stage of modern cameras, in addition to better image quality, and new trends of miniaturization are addressed in spectrometry [5] and imaging systems [6].

Curving the detector is an original technological challenge. Three strategies have been demonstrated in literature. The first one consists of adapting microtechnologies to curved substrates $[7,8]$; nevertheless, the process is too complicated and optical resolution is not sufficient enough to address today's microelectronics constraints. The second solution is based on a dedicated architecture realized either on polymer [9] or silicon-oninsulator wafers [10]: the detector is structured in small separated islands of devices interconnected by metallic lines. These devices could be twisted and flexed [11], and address applications such as paperlike displays, electronic skin, and biomedical devices. The specific hemispherical shape is less focused in literature, but two main references $[\underline{10}, \underline{12}]$ exist, and the most advanced [12] achieved a hemispherical eye camera with a $16 \times 16$ pixel array. However, all these solutions require a specific device fabrication process, which means that different technological steps must be made to realize the device, and the fill factor is decreased due to the architecture needed to bend. The last solution relies on thinning, partially or entirely, the component $[13,14]$. Because of the a priori on the fragility of such a structure, this solution is less discussed and no functional device has been presented so far.

The process developed and presented here is compatible with device manufacturing, considering minor modifications and low cost. Indeed, this work has been developed with off-the-shelf components, so the design is not specific to our purpose. The bending ability is given by an added step: the thinning, performed at the end of the fabrication process. The total thickness is decreased to $50 \mu \mathrm{m}$, which is now a standard in microelectronics [15]. By thinning, the whole device reaches e sufficient flexibility to stretch the detector into a hemispherical shape. Strain in this mechanical deformation is mainly proportional to the ratio between the device thickness and the radius of curvature. First mechanical results performed on square silicon samples show that the average strain is inferior to the silicon yield stress, which is about 4 to $9 \mathrm{GPa}$ depending the thinning process (with or without stress relief) [16]. For instance, the strain of a 50- $\mu \mathrm{m}$ thick silicon sample curved to a radius of $45 \mathrm{~mm}$ is $209 \mathrm{MPa}$. After thinning, the device is curved on a holder that is concave, convex, or even cylindrical, and placed underneath the device. Presently, the holder is a spherical lens cut in a square, but a dedicated electrical board could be spherically machined for future prototypes. By applying a mechanical force through a soft tool in contact with the thinned detector, the component is bent to fit the holder surface underneath. The component is free to deform, except the four corners, which are simply in contact on the holder. The soft tool is progressively deformed onto the component by the force applied in fitting the holder shape. This curved deformation is kept by 
curing an underfilling layer between the holder and the component. Once the curing step is done, the component is definitively curved onto the holder, reproducing precisely the shape targeted. In this way, we curved a conventional microbolometer glued onto a holder, which has been interconnected on a standard electrical board.

This process can be applied to any kind of devices, either bulk material or functional imagers: complementary metal-oxide semiconductor (CMOS), CCD, cooled photovoltaic IR detectors based on HgCdTe, or uncooled IR microbolometers. As silicon is the main material of these devices, first experiments have been made on thinned silicon samples with chemical mechanical polishing stress relief. The minimum radii achieved is $40 \mathrm{~mm}$. In this Letter, the process has been performed on bolometers. These detectors are composed of a readout circuit (CMOS device) above which the detecting matrix is processed. This is an array of small rectangular membranes formed by an absorbing layer in amorphous silicon; they are supported by arms that create a cavity up to the readout circuit. IR radiation is absorbed by the membrane, and converted into a temperature rise. This creates a change in the electrical resistance of the membrane, which is collected by the readout circuit. From a manufacturing point of view, a sacrificial layer is deposited on the readout circuit to pattern the silicon membrane. The plasma release of this temporary layer is made as a final step, just before housing the detector in the package.

The IR detector used to realize the hemispherical camera is a standard $320 \times 256$ pixel array at $25 \mu \mathrm{m}$ pitch. The holder is a concave glass holder scaled at the device size. It is an initial choice to realize the first prototype of an IR camera as achieved here. A dedicated holder could be achieved by machining the electrical board with a curved imprint for the detector; this solution will be used for the next cameras.

The process applied to this functional detector is the following: we use an off-the-shelf microbolometer with the sacrificial layer. The substrate is thinned down to $50 \mu \mathrm{m}$. An intermediate electrical test has been performed on this thinned device in order to check that this added step did not damage the electrical functionality; the result will be discussed later. Then, by applying a force, the device is curved on a concave holder and the curvature is kept by the underfill (epoxy glue). Once the shape is achieved, the usual bolometer fabrication process is pursued: plasma release is performed to remove the sacrificial layer under the pixels, and the component is electrically connected to the usual board.

The curvature performance is described in Fig. 1. The curved component is scanned by an optical profilometer. The radius of curvature is calculated from the perfect spherical shape deduced from the scanned area. The device radius is $73 \mathrm{~mm}$. This radius is larger than the one achieved in the experiments on silicon samples; this is mainly due to the multiple layer of different materials composing the functional devices, and the grinding process that was performed on single devices, without stress relief, which is available only on wafers. The holder radius is $70 \mathrm{~mm}$. The radius difference between the holder and the microbolometer could be explained by the underfill thickness, which is not presently homogeneous through the area, and the defects of the holder, which is not

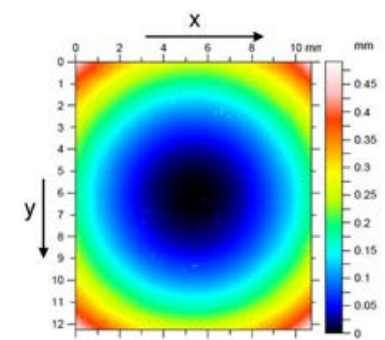

(a)

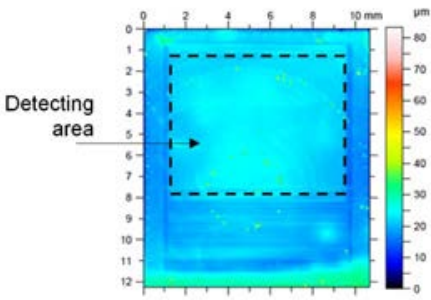

(b)
Fig. 1. (Color online) Images of the curved detector array scanned by optical profilometry, the colored scale illustrates the deformation in the $z$ axis. (a) is the scanned surface, and (b) is from the software analysis; it represents defects from the scanned surface to the perfect sphere.

perfectly spherical. Both parameters should be suppressed by further process optimizations. However, the perfect spherical surface is really close to the scanned surface, as shown in Fig. 1(b). It represents the defects from the scanned surface [Fig. 1(a)] to the perfect sphere. We can easily distinguish in this figure the structure of the FPA. Height variation through the device is $5 \mu \mathrm{m}$, so the curvature is well spherical and no undesired folds appear.

This curved device [Fig. 2(a)] is easily bonded on the electrical board by conventional wire bonding [Fig. 2(b)] and placed in a conventional thermal vacuum chamber. The final component is characterized as a conventional device. We are then able to achieve an electrical test and capture our very first picture with this new type of device configuration.

Electrical characterizations have been made after each crucial step: thinning and curvature. Indeed, both steps induce strains in the device that could deteriorate the electrical performance (modified bandgap structure, carrier mobility). The electrical parameters measured (see Table 1) are represented as a variation from the thinned and curved step compared to the bulk device.

Voltage variation between bulk and thinned is less than $1 \%$, and between bulk and curved is less than the dispersion in measures realized on the same bulk devices (e.g., $6 \%$ at $30{ }^{\circ} \mathrm{C}$ ). Noise is quite sensitive to measures: measurement dispersion is high (theoretically about 5\%); nevertheless, the noise measured after curvature is smaller. The signal response is really close to the bulk value in the range of deviation accepted in industry, e.g., 1.5\% [17]. So the thinning and the curvature did not damage the component performance.

To go further, we made a camera inspired by the human eye. The single lens is aspherical and plays the role

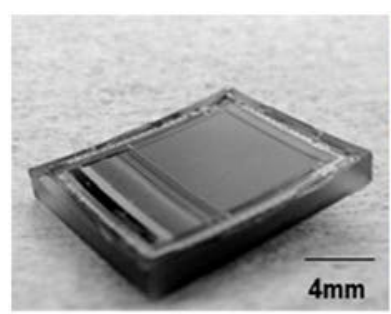

(a)

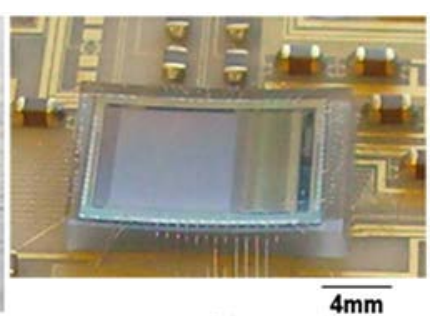

(b)
Fig. 2. (Color online) Pictures of curved microbolometer: (a) the thinned curved component on a glass holder; (b) this curved bolometer bonded onto an electrical board. 
Table 1. Electrical Characterization of Thinned and Curved Bolometer ${ }^{a}$

\begin{tabular}{cccccc}
\hline & \multicolumn{2}{c}{ Reference } & & \multicolumn{2}{c}{ Blackbody at $30^{\circ}$} \\
\cline { 2 - 3 } \cline { 5 - 6 } & Voltage & Noise & & Voltage & Noise \\
\hline$\Delta \mid$ thinned-bulk $\mid$ & $0.4 \%$ & $4 \%$ & - & - \\
$\Delta \mid$ curved-bulk $\mid$ & $0.2 \%$ & $4.6 \%$ & $5.8 \%$ & $2.7 \%$ \\
& Signal response & NETD & & \\
Bulk bolometer & $6.19 \mathrm{mV} / \mathrm{K}$ & $68 \mathrm{mK}$ & - & - \\
Curved bolometer & $6.25 \mathrm{mV} / \mathrm{K}$ & $59 \mathrm{mK}$ & - & - \\
\hline
\end{tabular}

${ }^{a}$ Parameters measured are the voltage and the noise measured at the reference stage, that is, when the device is not detecting IR radiation, and when the device sees a blackbody at $300 \mathrm{~K}$. The thinned bolometer is characterized only at the reference stage, because it still has the sacrificial layer, so pixels are not functional. Signal response is the voltage difference for a small temperature change. NETD is the noise equivalent temperature difference representing the sensitivity of the detector to thermal radiation.

of the cornea, and the detector is curved like the retina. Figure 3(a) presents the optical system: the focal length is $12.4 \mathrm{~mm}$, the field of view is $60^{\circ}$, and the $f$-number is 1.8. The overall system is $27.4 \mathrm{~mm}$ long. The main aberrations of this optical system are distortion and field curvature, which is suppressed by the curved focal plane, and leads to good resolution. Considering a similar planar system, such an image quality would not be possible, except by adding one more lens.

The system performance has been measured by bar targets at different spacings. The resulting modulation transfer function (MTF) in Fig. 3(b), shows good uniformity between on- and off-axis responses. Images captured by this bioinspired system [Fig. 3(c)] are well resolved and have good contrast. This picture proved

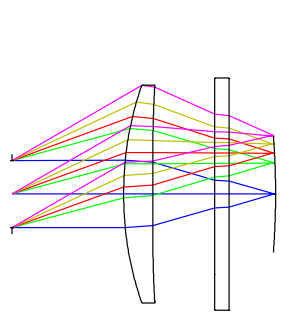

(a)

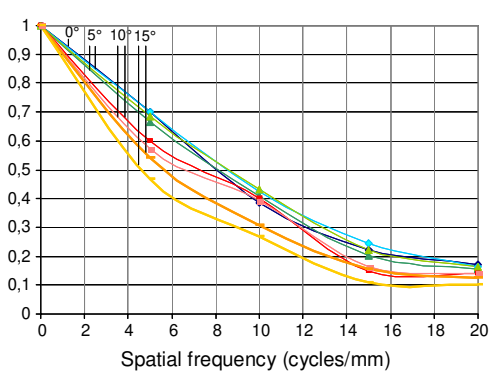

(a)

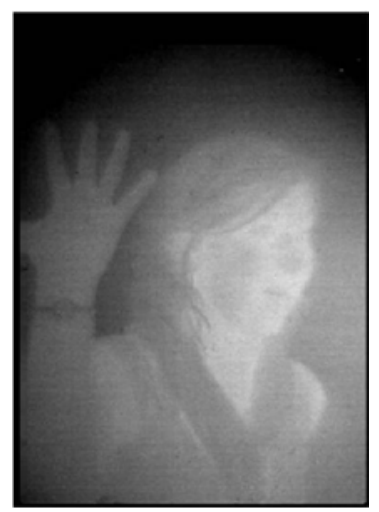

(c)

Fig. 3. (Color online) IR camera inspired by the human eye, system $f /=1.8$ and focal length $12.4 \mathrm{~mm}$. (a) Optical system; (b) experimental MTF; (c) IR picture captured by this camera. the good functionality of the curved detector on its whole surface; this image quality would not be possible with a planar FPA.

In conclusion, we developed a process to curve into a hemispherical shape a monolithic $320 \times 256$ pixel detector; the unique added step of thinning is easily achievable in the device fabrication process and the curvature is done using a dedicated holder. The final device is mounted and characterized like usual planar devices. A functional camera based on the human eye, with a curved IR bolometer and a unique lens, has been made without any electrical damage. Images in this biomimetic configuration have been obtained. They represent the very first pictures achieved in the state of the art by a monolithic curved detector, to the best of our knowledge.

By this work, we have initiated a new kind of device that simplifies optical designs. This result can be transferred to any kind of detectors. Opportunities offered by this new curved design are wide especially for miniaturized applications such as spectrometers [10] and imaging systems [11].

This work has been realized thanks to J. B. Moullec and the French Ministry of Army (DGA). The authors thank G. Gaude for the thinning step, J. Routin, and A. Gueugnot, E. de Borniol and A. Perez for the electrical and optical tests.

\section{References}

1. F. de la de la Barriere, G. Druart, N. Guerineau, and J. Taboury, Appl. Opt. 50, 943 (2011).

2. A. Brückner, J. Duparré, R. Leitel, P. Dannberg, A. Braüer, and A. Tünnermann, Opt. Express 18, 24379 (2010).

3. G. Muyo, A. Singh, M. Andersson, D. Huckridge, A. Wood, and A. R. Harvey, Opt. Express 17, 23, 21118 (2009).

4. S.-B. Rim, P. B. Catrysse, R. Dinyari, K. Huang, and P. Peumans, Opt. Express 16, 4965 (2008).

5. I. Avrutsky, K. Chaganti, I. Salakhutdinov, and G. Auner, Appl. Opt. 45, 7811 (2006).

6. G. Druart, N. Guérineau, R. Haidar, S. Thétas, J. Taboury, S. Rommeluère, J. Primot, and M. Fendler, Appl. Opt. 48, 3368 (2009).

7. S. Snow and S. C. Jacobsen, Microelectron. Eng. 84, 11 (2007).

8. Z. Li, Y. Gu, L. Wang, H. Ge, W. Wu, Q. Xia, C. Yuan, Y. Chen, B. Cui, and R. S. Williams, Nano Lett. 9, 2306 (2009).

9. D.-H. Kim, J.-H. Ahn, W. M. Choi, H.-S. Kim, T.-H. Kim, J. Song, Y. Y. Huang, Z. Liu, C. Lu, and J. A. Rogers, Science 320, 507 (2008).

10. R. Dinyari, S.-B. Rim, K. Huang, P. B. Catrysse, and P. Peumans, Appl. Phys. Lett. 92, 091114 (2008).

11. J. A. Rogers, T. Someya, and Y. Huang, Science 327, 1603 (2010).

12. H. C. Ko, M. P. Stoykovich, J. Song, V. Malyarchuk, W. M. Choi, C.-J. Yu, J. B. Geddes 3rd., J. Xiao, S. Wang, Y. Huang, and J. A. Rogers, Nature 454, 748 (2008).

13. P. Swain, Proc. SPIE 5499, 281 (2004).

14. S. Nikzad, T. J. Jones, and M. E. Hoenk, "Curved focal plane arrays for compact, wide field-of-view optical systems," presented at the 38th Lunar and Planetary Science Conference, League City, Texas, March 12-16, 2007.

15. R. Tummala, System on Package: Miniaturization of the Entire System, 1st ed. (McGraw-Hill Professional, 2008).

16. S. Johansson, F. Ericson, and J.-Å. Schweitz, J. Appl. Phys. 65, 122 (1989).

17. "ULIS," http://www.ulis-ir.com. 